\title{
Ratiometric Single-Nanoparticle Oxygen Sensors for Biological Imaging*
}

\author{
Changfeng Wu, Barbara Bull, Kenneth Christensen, and Jason McNeill \\ Department of Chemistry, Clemson University, Clemson, SC 29634 (USA), Fax: (+1) 864-656-6613, \\ E-mail: mcneill@clemson.edu
}

\section{Keywords}

fluorescent probes; FRET; nanostructures; polymers; sensors

\begin{abstract}
Oxygen is a critical component for many physiological and pathological processes in living cells. [1,2] Tissue hypoxia has been found to be closely related to the clinical course of a variety of diseases,[3] such as tumor growth,[4,5] diabetic retinopathy,[6] and rheumatoid arthritis. [7] Therefore, the measurement and imaging of oxygen levels in live cells and tissue represent a challenging and significant problem in modern biology, physiology, and medicine. Many efforts have been focused on the development of optical sensors for oxygen.[8-11] Oxygen sensing by phosphorescence quenching is noninvasive, sensitive, selective for oxygen, and can be implemented for real-time measurements as well as high-resolution oxygen mapping in tissue.[12-15] While conventional phosphorescent dyes for oxygen sensing are typically based on organometallic complexes and metalloporphyrins,[16,17] efforts to synthesize new chromophores with improved characteristics, $[18,19]$ or to modify them for applications such as multiphoton microscopy have also been reported.[20]
\end{abstract}

There is currently considerable interest in the development of nanoparticle-based optical oxygen sensors.[12,13,21] Such sensors typically consist of phosphorescent dyes encapsulated inside a polymer or silica nanoparticle, which serves to isolate the dyes from the cellular environment.[13] Moreover, nanoparticles typically exhibit higher brightness and better photostability than molecular dyes, owing to large numbers of chromophores per particle as well as the protective matrix. We recently demonstrated that $\pi$-conjugated polymer nanoparticles (CPdots) exhibit extraordinarily high fluorescence brightness under both onephoton and two-photon excitation.[22-24] Dye-doped conjugated polymer nanoparticles were also found to sensitize bright emission from fluorescent dye dopants by efficient energy transfer from the polymer to the dye.[25,26] Herein, we describe a novel nanoparticle architecture for oxygen sensing that consists of $\pi$-conjugated polymer molecules doped with an oxygensensitive phosphorescent dye. Upon light excitation, the polymer efficiently transfers energy to the phosphorescent dye, which results in bright phosphorescence that is highly sensitive to the concentration of dissolved oxygen. The salient features of the nanoparticle sensors, including their small size, extraordinary brightness, and ratiometric emission, together with the demonstration of single-particle sensing and cellular uptake, indicate their excellent potential for quantitative imaging of local molecular oxygen concentration in living cells and tissues.

\footnotetext{
**We gratefully acknowledge financial support from the NSF/EPSCoR under grant nos. 2001RII-EPS-0132573 and 2004RIIEPS-0447660, NSF CAREER grant no. CHE-0547846, NIH grant no. 1R01M081040, and NSF EFRI grant no. ENG-0736007. 
The conjugated polymers employed as the doping host are the polyfluorene derivatives poly (9,9-dihexylfluorene) (PDHF) and poly(9,9-dioctylfluorene) (PFO). Platinum(II) octaethylporphine (PtOEP) served as the oxygen sensitive dye (structures are shown in Figure 1a). Rapid addition of a solution of polyfluorene and PtOEP in THF to water, followed by mixing, led to the collapse of polymer chains because of the sudden increase in the THF/water ratio, which results in nanoparticle formation and simultaneous entrapment of the hydrophobic PtOEP molecules inside the nanoparticles (see the Experimental Section). The AFM results for PDHF-based particles indicate that the resulting particles are approximately spherical in shape, with particle heights (diameters) in the range of $(25 \pm 5) \mathrm{nm}$ (Figure $1 \mathrm{~b}, \mathrm{c})$. It was found that the preparation conditions for PFO-based particles, (in particular, the THF/water ratio) affected the polymer phase (glassy phase versus $\beta$ phase) in the resulting nanoparticles, and the polymer phase has a strong effect on the efficiency of energy transfer to the dye dopants. [27] In agreement with previous results, the glassy PFO phase particles were found to exhibit more efficient energy transfer to PtOEP than the $\beta$-phase PFO particles. The $10 \mathrm{wt} \%$ PtOEPdoped $\mathrm{PFO}$ particles also exhibit roughly spherical morphology, with particle sizes in the range $(50 \pm 10) \mathrm{nm}$.

Aqueous dispersions of the PtOEP-doped CPdots are clear and stable, with a faint pink color that arises from the visible absorption peak of PtOEP at $534 \mathrm{~nm}$ (Figure 2a, inset). Our previous results indicated that the ratio of dye to polymer in the CPdots is similar to that of the precursor solution, with no appreciable dye leakage.[26] UV/Vis absorption spectra (Figure 2a) are consistent with the dye/polymer weight ratio (1:10) of the precursor solution, and the absorption cross-sections (ca. $380 \mathrm{~nm}$ ) of PtOEP-doped PDHF and PFO nanoparticles in water were determined to be approximately $1.9 \times 10^{-12} \mathrm{~cm}^{2}$ and $1.2 \times 10^{-11} \mathrm{~cm}^{2}$, respectively. According to the particle size and the dye/polymer weight ratio, it is estimated that each doped PDHF particle (ca. $25 \mathrm{~nm}$ diameter) consists of about 90 PDHF molecules and about 700 PtOEP molecules. Both PDHF and PtOEP contribute to the nanoparticle absorption at $380 \mathrm{~nm}$, and their relative contributions are estimated to be around 80\% from PDHF and around 20\% from PtOEP, respectively. Similarly, each doped PFO particle (ca. $50 \mathrm{~nm}$ diameter) contains approximately 270 PFO molecules and approximately 5500 PtOEP molecules. The large absorption cross-sections of the CPdots (roughly 20 times higher than those of dye-loaded silica or polymer particles of similar dimensions) provides a clear indication of the potential brightness advantage of the polyfluorene-based particles.

The Förster radius of the donor-acceptor pair, which characterizes the efficiency of energy transfer between the PFO donor and the PtOEP acceptor, was calculated to be approximately $1.7 \mathrm{~nm}$, which is rather small because of the nonoptimal spectral overlap (Figure 2b). However, exciton diffusion in the conjugated polymer host results in efficient energy transfer, even at modest doping levels. By employing our previously developed random walk model for estimating energy transfer efficiency in dye-doped conjugated polymer nanoparticles,[26] the calculated energy transfer efficiency for 10\% PtOEP-doped PFO nanoparticles (ca. $50 \mathrm{~nm}$ ) was approximately $89 \%$, which is in good agreement with the experimental value (ca. 87\%) obtained from fluorescence spectra (Figure 2c). Time-resolved fluorescence measurements were performed to provide information about the rate of energy transfer within the nanoparticles (Figure 2d). A fluorescence lifetime of $110 \mathrm{ps}$ was obtained from the decay curves of the undoped PFO dot emission at $420 \mathrm{~nm}$, and the lifetime was reduced to $18 \mathrm{ps}$ for the $10 \%$ PtOEP-doped PFO dots, primarily because of energy transfer to the dopant. The energy transfer rate constant $\left(k_{\mathrm{ET}}\right)$ was deduced by subtracting the decay rate constant of undoped dots $\left(\tau_{\mathrm{D}}^{-1}=9 \mathrm{~ns}^{-1}\right)$ from the total decay rate constant of the doped nanoparticles $\left(\tau_{\mathrm{D}}^{-1}=56 \mathrm{~ns}^{-1}\right)$. The result $\left(k_{\mathrm{ET}}=47 \mathrm{~ns}^{-1}\right)$ is in good agreement with the value obtained from analysis of the fluorescence spectra and the predictions of the nanoparticle energy transfer model. Irradiation of aqueous nanoparticle dispersions with a UV lamp $(365 \mathrm{~nm})$ illustrates a clear difference in 
the fluorescence color of undoped versus doped PFO nanoparticles (Figure 2c, inset). The emission spectra shown in Figure 2c were obtained at an excitation wavelength of $350 \mathrm{~nm}$ where the absorption of PtOEP is negligible. When compared to the undoped PFO dots, the doped dots exhibited significantly reduced PFO fluorescence and strong red emission $(650 \mathrm{~nm})$ from PtOEP, which is consistent with energy-transfer-mediated phosphorescence in the doped particles. The phosphorescence quantum yield of the PtOEP-doped PFO dots (nitrogensaturated solution) was determined to be approximately $9 \%$ (a dilute solution of tetraphenylporphyrin in toluene was used as a standard). This result is somewhat lower than that of PtOEP doped PFO thin film devices (ca. 20\%),[28] and is probably because of the higher doping concentration in the nanoparticles, which leads to increased self-quenching. The nanoparticle phosphorescence brightness, which is defined as the product of the optical cross section and the phosphorescence quantum yield, is more than 1000 times higher than that of conventional oxygen sensing dyes, and is estimated to be roughly 5-10 times higher than that of PtOEP-doped silica particles of similar dimensions at similar dye loading. The enhanced brightness is attributable to the combination of efficient light harvesting by the polymer and efficient energy transfer to PtOEP.

Oxygen-sensitive phosphorescence is readily observed from aqueous PtOEP-doped CPdot suspensions with different concentrations of dissolved oxygen (Figure 3). The nitrogensaturated CPdot suspension exhibits intense red emission, while the air- and oxygen-saturated samples present weaker emission because of oxygen quenching. The emission spectra (Figure 3a) exhibit a moderate fluorescence (ca. $420 \mathrm{~nm}$ ) from the PDHF host and oxygen-dependent phosphorescence (ca. $650 \mathrm{~nm}$ ) from PtOEP dopant. Significantly, the residual fluorescence from the donor (ca. $420 \mathrm{~nm}$ ) remains almost constant, while the acceptor phosphorescence (ca. $650 \mathrm{~nm}$ ) is highly sensitive to oxygen. This property facilitates ratiometric sensing, which is useful for applications such as cellular and tissue imaging of oxygen concentration, since the ratio of acceptor to donor fluorescence is relatively insensitive to the local nanoparticle concentration. By defining $R$ as the ratio of the emission intensity of the acceptor units (sensing dye) to that of the donor units (polymer reference), the sensitivity of the sensor can be expressed by the overall quenching response to dissolved oxygen [Eq. 1],[12,13]

$$
Q=\left(R_{\mathrm{N}_{2}}-R_{\mathrm{O}_{2}}\right) / R_{\mathrm{N}_{2}}
$$

where $R_{\mathrm{N}_{2}}$ and $R_{\mathrm{O}_{2}}$ represent the emission intensity ratios of the sensor in fully deoxygenated and fully oxygenated solutions, respectively. The measured $Q$ value for the doped CPdot particles is around $95 \%$, which is among the most sensitive phosphorescent oxygen-sensing nanoparticles reported to date.[12] Phosphorescence lifetime measurements provide an alternative method for quantitative oxygen sensing.[29] A comparison of the phosphorescence decays of the 10\% PtOEP-doped PDHF dots in nitrogen, air, and oxygen-saturated solutions is shown in Figure 3b. Each decay curve exhibits single-exponential decay kinetics, which indicates a homogeneous distribution of the PtOEP molecules inside the nanoparticles. A phosphorescence lifetime of approximately $37 \mu$ s was obtained for the nitrogen-saturated nanoparticle dispersion, which is consistent with the lifetime results (30-50 $\mu \mathrm{s})$ of PtOEP in a variety of organic thin-film devices.[28,30] The phosphorescence lifetime decreases to around $10 \mu$ s for the air-saturated nanoparticle solution, and to around $5 \mu$ s for the fully oxygenated solution, which indicates efficient quenching of phosphorescence by molecular oxygen. Quenching by molecular oxygen is rapidly and completely reversed by subsequent bubbling of $\mathrm{N}_{2}$ through the nanoparticle suspension.

The large optical cross-section, bright phosphorescence, and high oxygen sensitivity of the doped CPdots show great potential for mapping oxygen concentration in biological systems. 
The relatively small particle size (ca. $25 \mathrm{~nm}$ ) is advantageous for cellular uptake and distribution compared to other particle sensors, which are typically much larger.[12,13] The small size also provides a large surface-to-volume ratio and a reduced distance over which the oxygen must diffuse to reach the dyes, which should lead to excellent response time. The sensitivity, selectivity, and response time also depend on the doping matrix, in this case the conjugated polymer. Polyfluorenes such as PDHF and PFO are hydrophobic glassy polymers, which should exhibit good oxygen permeability while serving as a barrier to interfering ionic species. In a qualitative experiment, a dispersion of nitrogen-saturated CPdots was excited by using a UV lamp (365 nm). The bright phosphorescence was observed to dim within a few seconds upon exposure to air, which is a qualitative indication of adequate oxygen permeability and response time for a wide variety of applications. Fluorescence spectra and fluorescence quantum yields of CPdots suspended in a series of phosphate buffers with $\mathrm{pH}$ values ranging from 5 to 8 were indistinguishable from those of particles suspended in deionized water, thus indicating no apparent sensitivity to $\mathrm{pH}$ over this range. Additionally, we recently determined that CPdots exhibit extraordinarily large cross-sections for two-photon excitation (as high as $10^{5} \mathrm{GM}$ ),[23] which suggests that the nanoparticles are promising for 3D oxygen mapping in tissue by using two-photon-based imaging techniques.[20]

As a further test of the brightness and sensing capabilities of the CPdot particles, single-particle phosphorescence imaging was performed. Single-molecule detection of triplet emission in transition-metal complexes represents a challenging task because of the typically low phosphorescence quantum yields and very low radiative rates.[31-33] The PtOEP-doped CPdots are expected to present substantially brighter phosphorescence than that of single phosphorescent molecules because of the large number of phosphorescent chromophores per particle (ca. 700 phosphorescent chromophores in a particle of ca. $25 \mathrm{~nm}$ diameter). However, the excitation intensity must be carefully controlled in the case of the PtOEP-doped nanoparticles, since phosphorescence from the doped CPdots is readily saturated under high excitation intensity, while the fluorescence of the donor is less susceptible to saturation effects, which results in donor polymer emission that greatly exceeds that of the PtOEP, as well as reduced sensitivity of the PtOEP emission to oxygen (data not shown). Single doped PDHF dots (particle size $\approx 25 \mathrm{~nm}$ ) were immobilized on a glass coverslip and imaged using a custom built wide-field epifluorescence microscope. The $405 \mathrm{~nm}$ excitation laser was attenuated to an intensity of about $25 \mathrm{mWcm}^{-2}$ in the center of the laser spot in the sample plane, corresponding to roughly $5 \times 10^{4}$ photons absorbed per nanoparticle per second, which is well below saturation. Single-particle phosphorescence images of the CPdots under flowing nitrogen and in air were obtained (Figure 4a). Each peak in the image corresponds to a single doped CPdot, which exhibits a near-diffraction-limited Airy disk with a full width at half maximum value of approximately $300 \mathrm{~nm}$. AFM images of the same sample indicated similar particle densities, which provided further indication that the fluorescence features correspond to individual nanoparticles. Phosphorescence from a single CPdot particle on the coverslip exhibits sensitivity to oxygen content in the atmosphere above the coverslip. The phosphorescence intensity that corresponds to a single particle in an air atmosphere clearly shows that the phosphorescence is substantially quenched by oxygen. Subsequent imaging on the sample after resuming the nitrogen flow shows near-complete recovery of the phosphorescence intensity (Figure 4a left). The intensity changes were observed over several nitrogen-air cycles, with no apparent photobleaching, owing to the low excitation intensity employed. Some particleto-particle variability in oxygen sensitivity was observed, perhaps because of defects or blinking phenomena that occur in some of the nanoparticles. The single-particle oxygen sensing results provide clear indication that the nanoparticles are sufficiently bright and sensitive for a wide range of imaging and sensing applications.

The cellular uptake of CPdots was evaluated by incubating J774A1 cells (a macrophage-like murine cell line) that had been incubated with these doped CPdots (ca. $10 \mathrm{pM}$ ) for 10 hours in 
media (see the Experimental Section). A comparison of the differential interference contrast (DIC) image and phosphorescence images of the nanoparticle-labeled cells clearly indicates uptake of the CPdots (Figure 4b). The nanoparticle sensors do not appear to exhibit appreciable cytotoxicity or phototoxicity for the incubation and imaging conditions employed. A detailed understanding of the nanoparticle uptake mechanism as well as subcellular localization requires further investigation. In addition, the sensing of oxygen concentration in specific subcellular structures will require targeting of the nanoparticles by encapsulation and bioconjugation.

Nevertheless, the high brightness of the nanoparticles at low loading levels and low excitation levels, together with the facile uptake by cells, is promising for applications such as the determination of oxygen concentration in tissues and subcellular structures.

In summary, we have reported energy-transfer-mediated phosphorescence from metalloporphyrin-doped polyfluorene nanoparticles and its application to biological oxygen sensing. The conjugated polymer nanoparticles possess an extraordinary light-harvesting ability compared to typical dye-loaded silica or polymer nanoparticles; this leads to nanoparticle absorption cross-sections exceeding $10^{-12} \mathrm{~cm}^{2}$ (for particles ca. $25 \mathrm{~nm}$ in diameter). Fluorescence spectroscopy and time-resolved fluorescence measurements indicate highly efficient energy transfer from the host polymer to the metalloporphyrin acceptors, which results in bright phosphorescence that is highly sensitive to the concentration of molecular oxygen. Single-particle phosphorescence imaging results indicated the observed phosphorescence intensity from single particles was sensitive to the partial pressure of oxygen. The small size, extraordinary brightness under conventional and two-photon excitation, and ratiometric emission of the nanoparticle sensor, together with the demonstration of singleparticle sensing and cellular uptake, indicate the potential of the doped CPdots for quantitative mapping of local molecular oxygen levels in living cells and tissue.

\section{Experimental Section}

The conjugated polymers PDHF (average $M_{\mathrm{W}} 55000$, polydispersity 2.7, ADS Dyes, Inc.), PFO (average $M_{\mathrm{W}} 147000$, polydispersity 3.0, ADS Dyes, Inc.) and PtOEP (Frontier Scientific, Inc.) were used as provided without further purification.

Preparation of PtOEP-doped polyfluorene nanoparticles: Polymer (10 mg) was dissolved in HPLC grade THF (10 g) by stirring overnight under an inert atmosphere.

Preparation of PtOEP-doped PDHF nanoparticles: A solution (2 mL) of PDHF (50 ppm) and PtOEP (5 ppm) in THF was added quickly to deionized water $(8 \mathrm{~mL})$ while sonicating the mixture, followed by an additional 10 seconds of sonication.

Preparation of PtOEP-doped PFO nanoparticles: A solution $(200 \mu \mathrm{L})$ of PDHF (500 ppm) and PtOEP $(50 \mathrm{ppm})$ was injected quickly into deionized water $(8 \mathrm{~mL})$ under sonication. The THF was removed by partial evaporation under reduced pressure, followed by filtration through a 0.2 micrometer filter to remove larger aggregates.

AFM measurements: One drop of the nanoparticle dispersion was evaporated on a freshly cleaved mica substrate. The surface topography was imaged with an Ambios Q250 multimode AFM in AC mode. UV/Vis absorption spectra were recorded with a Shimadzu UV-2101PC scanning spectrophotometer using $1 \mathrm{~cm}$ quartz cuvettes. Fluorescence spectra were recorded using a commercial fluorometer (Quantamaster, PTI, Inc.). Nitrogen or oxygen was bubbled through aqueous nanoparticle suspensions for $5 \mathrm{~min}$ prior to taking photographs or measuring the emission spectra. Fluorescence lifetimes of the polyfluorene donor were measured by using time-correlated single-photon counting technique (TCSPC). [24] The phosphorescence lifetime measurements were performed using a home-built photon counting spectrometer, as described in a previous report.[34] 
Single-particle imaging: A dilute suspension of 10\% PtOEP-doped PDHF dots (ca. $25 \mathrm{~nm}$ diameter) was immobilized on a cleaned microscope cover glass. Single-particle phosphorescence imaging was performed on a customized wide-field inverted epifluorescence microscope using a $405 \mathrm{~nm}$ diode laser as excitation source and a 100X oil-immersion objective. The laser excitation at the sample plane exhibited a Gaussian profile with full width at half maximum of around $5 \mu \mathrm{m}$. Typical laser intensities employed were approximately 25 $\mathrm{mWcm}^{-2}$ in the center of the laser spot in the sample plane. Phosphorescence from the doped CPdots was filtered by the combination of two $600 \mathrm{~nm}$ longpass filters and then focused onto a deep-cooled, back-illuminated frame transfer CCD camera (Princeton Instruments, PhotonMAX: 512B) to yield a pixel resolution of $105 \mathrm{nmpixel}^{-1}$. An acquisition time of 60 seconds was employed, which yielded approximately 1000 detected photons per nanoparticle. Nitrogen was flowed into an airtight chamber above the sample that could be readily removed to permit imaging in ambient air. An overall luminescence detection efficiency of 5\% was determined using nile red loaded polystyrene spheres (Invitrogen).

For cellular imaging, J774 A1 macrophages were plated onto $35 \mathrm{~mm}$ glass-bottom culture dishes at approximately $2 \times 10^{5}$ cells per dish and allowed to incubate at $37^{\circ} \mathrm{C}$ with $\mathrm{CO}_{2}(5 \%)$ overnight. The next day, sterile filtered nanoparticle dispersions $(300 \mu \mathrm{L}, \mathrm{ca} .100 \mathrm{pM})$ were added to cell media (DMEM $+10 \%$ heat-inactivated fetal bovine serum, $3 \mathrm{~mL}$ ) and allowed to incubate for $10 \mathrm{~h}$. The cells were then washed three times with warm Ringer's buffer (155 $\mathrm{mMNaCl}, 5 \mathrm{~mm} \mathrm{KCl}, 2 \mathrm{~mm} \mathrm{CaCl}, 1 \mathrm{~mm} \mathrm{MgCl} 2,2 \mathrm{~mm} \mathrm{NaH} \mathrm{PO}_{4}, 10 \mathrm{~mm}$ Hepes, and $10 \mathrm{mM}$ glucose, $\mathrm{pH}$ 7.2-7.4) before viewing. Fluorescence and DIC images of cells were collected using an Olympus IX70 inverted epifluorescence microscope equipped with a 300W Xenon arc lamp (380 nm excitation filter; Chroma), and $40 \times$ objective (UPlanApo, $0.90 \mathrm{NA}$ ). The phosphorescence signal from the CPdot-labeled cells was collected by the same objective lens, filtered by a $630 \mathrm{~nm}$ bandpass filter with $60 \mathrm{~nm}$ bandwidth (Chroma), and detected by a CCD camera (OrcaER; Hamamatsu).

\section{References}

1. Acker T, Acker H. J Exp Biol 2004;207:3171. [PubMed: 15299039]

2. Lopez-Barneo J, Pardal R, Ortega-Saenz P. Annu Rev Physiol 2001;63:259. [PubMed: 11181957]

3. Ikeda E. Pathol Int 2005;55:603. [PubMed: 16185289]

4. Höckel M, Schlenger K, Hockel S, Aral B, Schaffer U, Vaupel P. Int J Cancer 1998;79:365. [PubMed: 9699528]

5. Carmeliet P, Dor Y, Herbert JM, Fukumura D, Brusselmans K, Dewerchin M, Neeman M, Bono F, Abramovitch R, Maxwell P, Koch CJ, Ratcliffe P, Moons L, Jain RK, Collen D, Keshet E. Nature 1998;394:485. [PubMed: 9697772]

6. Frank RN. N Engl J Med 2004;350:48. [PubMed: 14702427]

7. Distler JHW, Wenger RH, Gassmann M, Kurowska M, Hirth A, Gay S, Distler O. Arthritis Rheum 2004;50:10. [PubMed: 14730595]

8. Demas JN, Degraff BA. Anal Chem 1991;63:829A.

9. Preininger C, Klimant I, Wolfbeis OS. Anal Chem 1994;66:1841.

10. Rosenzweig Z, Kopelman R. Anal Chem 1995;67:2650. [PubMed: 8849030]

11. McDonagh C, MacCraith BD, McEvoy AK. Anal Chem 1998;70:45.

12. Koo YEL, Cao YF, Kopelman R, Koo SM, Brasuel M, Philbert MA. Anal Chem 2004;76:2498. [PubMed: 15117189]

13. Xu H, Aylott JW, Kopelman R, Miller TJ, Philbert MA. Anal Chem 2001;73:4124. [PubMed: 11569801]

14. Rumsey WL, Vanderkooi JM, Wilson DF. Science 1988;241:1649. [PubMed: 3420417]

15. Vinogradov SA, Lo LW, Jenkins WT, Evans SM, Koch C, Wilson DF. Biophys J 1996;70:1609. [PubMed: 8785320] 
16. Carraway ER, Demas JN, Degraff BA, Bacon JR. Anal Chem 1991;63:337.

17. Mills A, Lepre A. Anal Chem 1997;69:4653.

18. Borisov SM, Klimant I. Anal Chem 2007;79:7501. [PubMed: 17718539]

19. Zhang G, Chen J, Payne SJ, Kooi SE, Demas JN, Fraser CL. J Am Chem Soc 2007;129:8942. [PubMed: 17608480]

20. Briñas RP, Troxler T, Hochstrasser RM, Vinogradov SA. J Am Chem Soc 2005; 127:11851. [PubMed: 16104764]

21. Clark HA, Hoyer M, Philbert MA, Kopelman R. Anal Chem 1999;71:4831. [PubMed: 10565274]

22. Wu C, Szymanski C, McNeill J. Langmuir 2006;22:2956. [PubMed: 16548540]

23. Wu C, Szymanski C, Cain Z, McNeill J. J Am Chem Soc 2007;129:12904. [PubMed: 17918941]

24. Wu C, Bull B, Szymanski C, Christensen K, McNeill J. ACS Nano 2008;2:2415. [PubMed: 19206410]

25. Wu C, Peng H, Jiang Y, McNeill J. J Phys Chem B 2006;110:14148. [PubMed: 16854113]

26. Wu C, Zheng Y, Szymanski C. J McNeill, J Phys Chem C 2008;112:1772.

27. Wu C, McNeill J. Langmuir 2008;24:5855. [PubMed: 18459748]

28. Lane PA, Palilis LC, O’Brien DF, Giebeler C, Cadby AJ, Lidzey DG, Campbell AJ, Blau W, Bradley DDC. Phys Rev B 2001;63:235206.

29. Lakowicz, JR. Principles of Fluorescence Spectroscopy. Vol. 3. Springer; Berlin: 2006.

30. Baldo MA, O’Brien DF, You Y, Shoustikov A, Sibley S, Thompson ME, Forrest SR. Nature 1998;395:151.

31. Mei EW, Vinogradov S, Hochstrasser RM. J Am Chem Soc 2003;125:13198. [PubMed: 14570495]

32. Vacha M, Koide Y, Kotani M, Sato H. Chem Phys Lett 2004;388:263.

33. Hu DH, Lu HP. J Phys Chem B 2005;109:9861. [PubMed: 16852191]

34. Peng H, Wu C, Jiang Y, Huang S, McNeill J. Langmuir 2007;23:1591. [PubMed: 17279632] 
a)

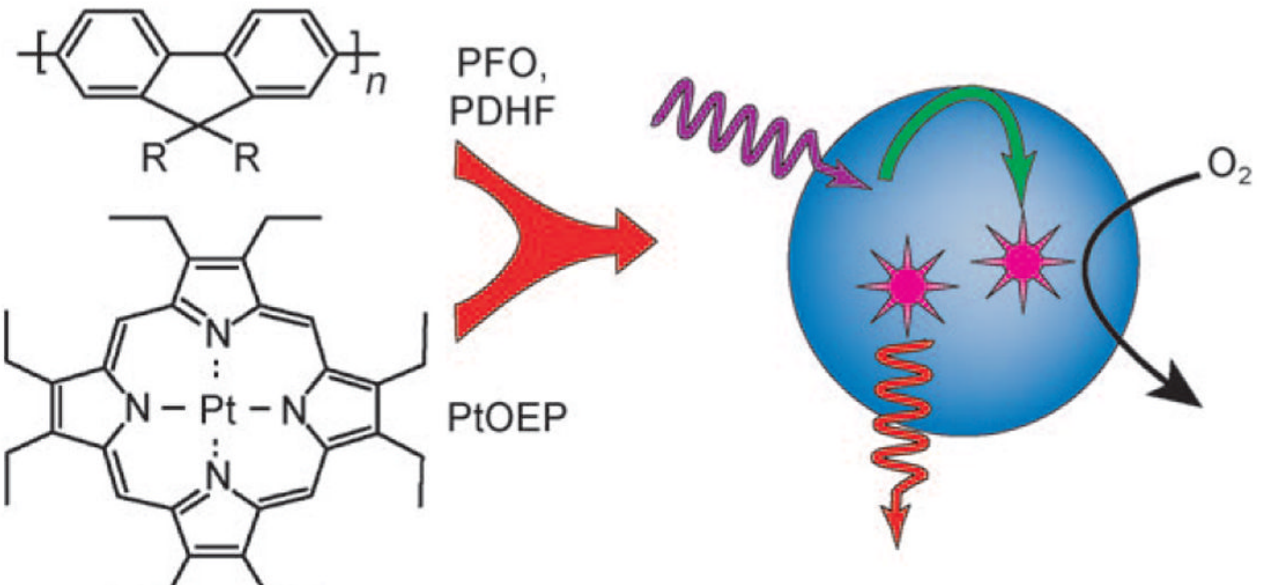

b)

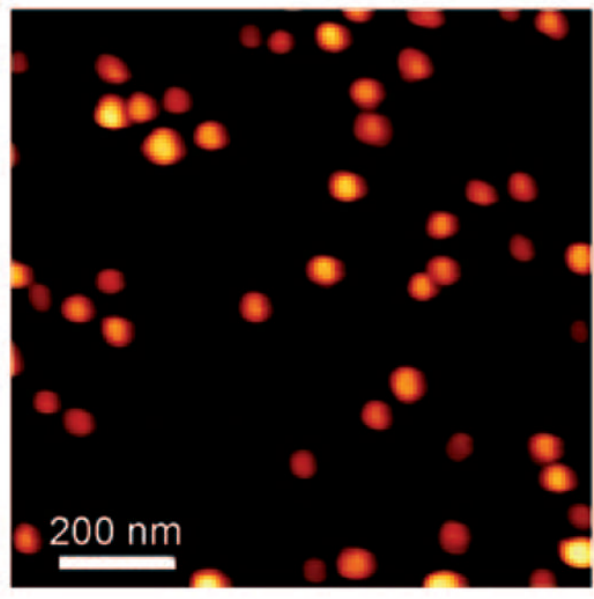

c)

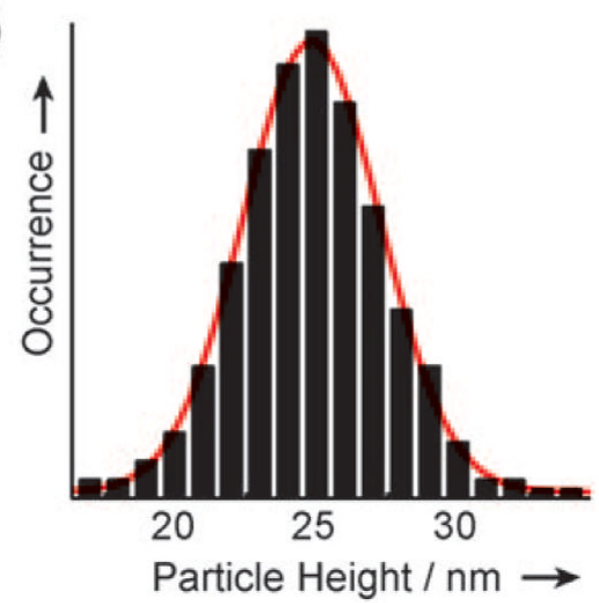

Figure 1.

a) Schematic illustration of the formation of conjugated polymer dots for oxygen sensing. b) Representative AFM image of PtOEP-doped PDHF dots dispersed on a mica substrate. c) Histogram of particle-height data obtained from the AFM image in (b). 

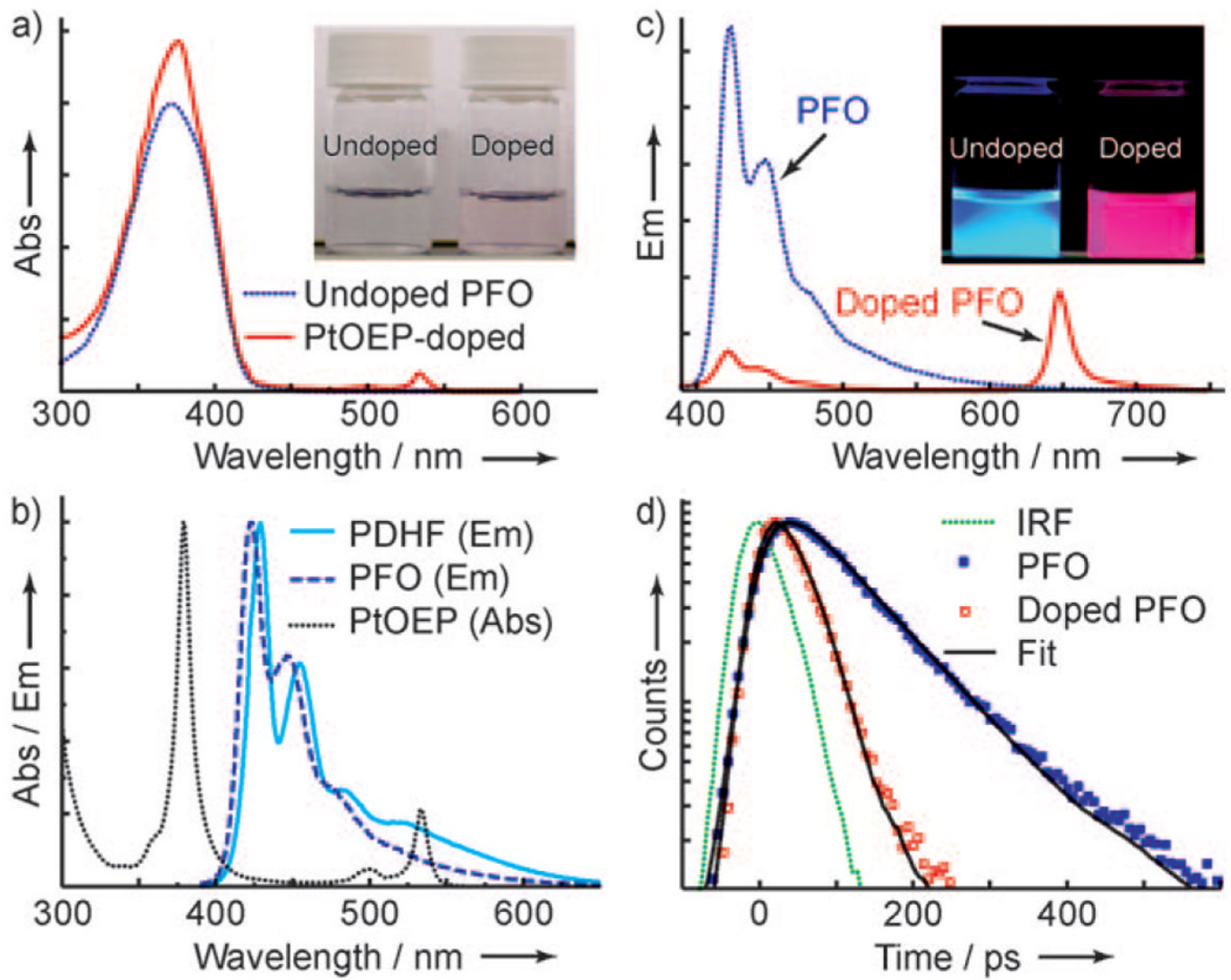

Figure 2.

a) UV/Vis absorption spectra of the undoped and PtOEP-doped PFO dots in water. The inset shows aqueous dispersions of PFO dots under room light. b) Spectral overlap between fluorescence emission of polyfluorene nanoparticles and absorption of the PtOEP dye. c) Emission spectra of the undoped and PtOEP-doped PFO nanoparticles with an excitation wavelength of $350 \mathrm{~nm}$. The inset shows aqueous dispersion of PFO dots under a UV lamp (365 $\mathrm{nm})$. d) Fluorescence decays of the undoped and PtOEP-doped PFO dots measured by a TCSPC setup. The green curve shows the instrumental response function (IRF). The scattered symbols represent experimental data, and the solid lines are fits. 

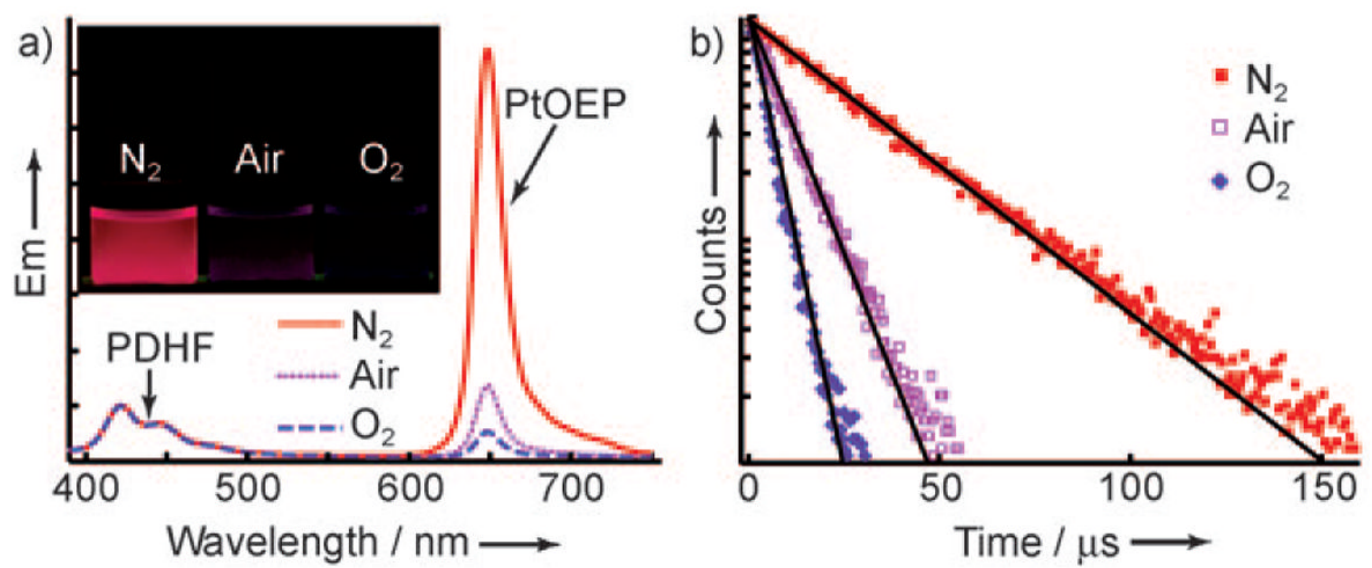

Figure 3.

a) Oxygen-dependent emission spectra of the 10\% PtOEP-doped PDHF dots (excitation wavelength $=350 \mathrm{~nm}$ ). The inset shows doped PDHF dots in aqueous solutions saturated with nitrogen, air, and oxygen, respectively, under a UV lamp. b) Phosphorescence decays of the $10 \%$ PtOEP-doped PDHF dots with different oxygen concentrations. The scattered symbols represent experimental data, and the solid lines are fits. 
a)
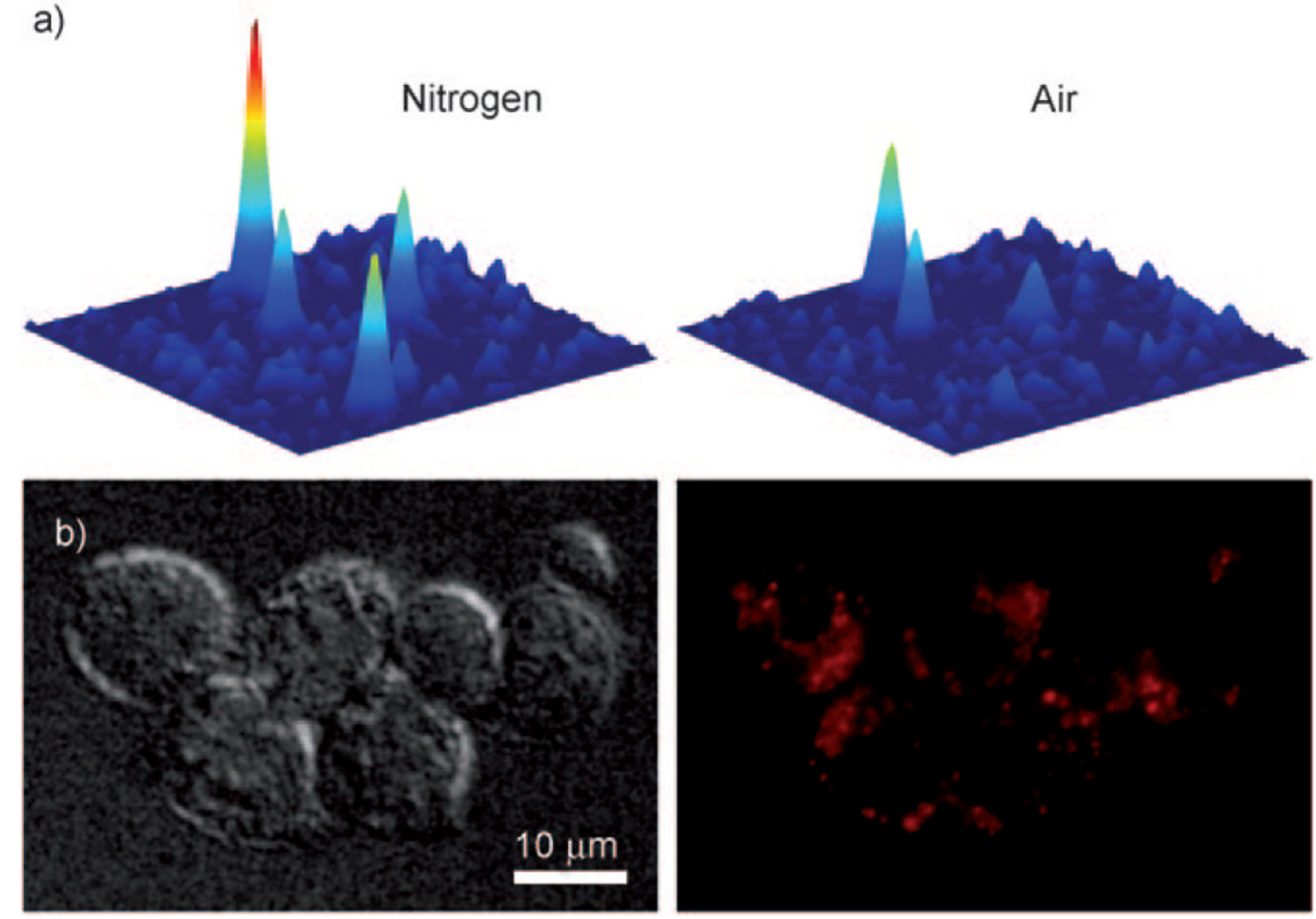

Figure 4.

a) Single-particle phosphorescence images of the doped CPdots immobilized on a coverslip under a nitrogen and an air atmosphere, respectively. b) DIC and phosphorescence images that indicate uptake of the nanoparticle sensor by macrophage cells. 